\title{
Temporal trends of $\beta$-haemolytic streptococcal osteoarticular infections in western Norway
}

\author{
Oddvar Oppegaard ${ }^{1,2 *}$, Steinar Skrede ${ }^{1,2}$, Haima Mylvaganam ${ }^{3}$ and Bård Reiakvam Kittang 2,4
}

\begin{abstract}
Background: Beta-haemolytic streptococci are important contributors to the global burden of osteoarticular infections (OAI). Knowledge on the disease traits specific for streptococcal OAl, however, remains scarce. We wished to explore temporal trends of OAI caused by Group A Streptococci (GAS), Group B Streptococci (GBS) and Group C and G Streptococci (GCGS), and furthermore, to describe the associated host and pathogen characteristics.

Methods: All cases of microbiologically verified $\beta$-haemolytic streptococcal OAl in Health Region Bergen, Norway, in the period 1999-2013 were retrospectively identified. Clinical data were extracted from medical records. Microbial isolates were submitted to antibiotic susceptibility testing and molecular typing.

Results: A total of 24 GAS, 45 GBS and 42 GCGS acute OAl were identified. The cumulative incidence of GCGS OAl, but not GAS or GBS OAl, increased significantly from the first to the last 5-year period (IRR 5.7, $p=0.0003$ ), with the annual incidence peaking at 1.9/100 000 in 2013. GAS OAl generally produced the most acute and severe clinical presentation, whereas GBS and GCGS predominantly affected the elderly, and were significantly associated with the presence of host risk factors of systemic and focal origin, respectively.

Conclusions: We found a significantly increasing incidence of GCGS OAl, likely related to the presence of host susceptibility factors, including prosthetic material and pre-existing joint disease. With an increasing application of therapeutic and diagnostic bone and joint procedures, the rising trend of OAI caused by GCGS is likely to continue. Sustained epidemiological attentiveness to GCGS seems warranted.
\end{abstract}

Keywords: Streptococcus dysgalactiae, Streptococcus pyogenes, Streptococcus agalactiae, Osteoarticular infection, Septic arthritis, Epidemiology

\section{Background}

Osteoarticular Infections (OAI) cause significant morbidity worldwide, and the incidence is increasing [1-4]. This likely reflects both a higher burden of comorbidity in the population, and an increased performance of surgical bone and joint procedures $[1,2]$.

Streptococci are consistently second only to staphylococci as causative agents of OAI, representing approximately $15-20 \%$ of the cases [4-6]. However, knowledge on clinical and microbial characteristics specific for streptococcal OAI is scarce, and mostly available from

\footnotetext{
* Correspondence: Oddvar.Oppegaard@helse-bergen.no

'Department of Medicine, Haukeland University Hospital, 5021 Bergen, Norway

${ }^{2}$ Department of Clinical Science, University of Bergen, Bergen, Norway Full list of author information is available at the end of the article
}

small case series published decades ago [7]. The few retrospective cohort studies published revealed a predominance of $\beta$-haemolytic streptococci, with group A Streptococci (GAS) and group B streptococci (GBS) comprising the majority [8-10]. Furthermore, speciesassociated clinical features were suggested; GBS mainly affecting the multi-morbid and elderly, and GAS producing more severe clinical manifestations. However, the number of included cases in these studies was generally too small to permit statistical analyses or evaluate temporal trends.

Streptococcus dysgalactiae subspecies equisimilis (SDSE), a $\beta$-haemolytic streptococcus predominantly possessing Lancefield group C or G antigen (GCS and GGS), has recently emerged as an important pathogen, 
increasingly associated with invasive disease [11]. We wished to explore if this rising trend was reflected in the rates of osteoarticular GCS and GGS-infections, and to describe the host and pathogen characteristics of these infections in comparison to OAI caused by GAS and GBS.

\section{Methods}

\section{Study setting}

Health Region Bergen, Western Norway, comprises the tertiary care hospital Haukeland University Hospital, and the two secondary care hospitals Haraldsplass Deaconess Hospital and Voss Hospital. The population in the catchment area increased from 360796 to 427486 inhabitants during the study period. The epidemiology of invasive $\beta$-haemolytic streptococcal disease within this region in the period 1999-2013 has previously been described [11]. Cases conforming to the OAI case definition were included, and demographic and clinical information was abstracted from medical records.

\section{Definitions}

Osteoarticular infections comprised Native Joint Infections (NJI), defined as Newman group A and B [12], Prosthetic Joint Infection (PJI), identified and classified according to IDSA guidelines [13], and Acute Osteomyelitis (AOM), inferred from a radiologically verified boneinfection in conjunction with a positive blood culture or bone biopsy obtained though intact skin. AOM included both peripheral and vertebral osteomyelitis. Cases with chronic osteomyelitis (symptom duration $>4$ weeks) or concurrent endocarditis, were excluded. Repeatedly positive cultures with the same organism identified within 30 days of initial isolation were considered to be from a single episode. Relapse was defined as a clinical deterioration after cessation of antibiotic treatment, of verified $\beta$-hamolytic streptococcal or unidentified microbiological aetiology, resulting in a new medical or surgical intervention. Referrals from other hospitals and patients with a registered permanent residence address outside the catchment area at the time of admission were excluded. Systemic inflammatory response syndrome (SIRS) and Streptococcal toxic shock syndrome (STSS) were defined as previously described [14, 15]. Sequelae were defined as presence of arthrosis, chronic joint pain, reduced joint motility, amputation or chronic infection treated with suppressive antibacterial therapy.

\section{Bacterial identification, typing and antibiotic susceptibility}

All isolates displayed large colony size $(>0.5 \mathrm{~mm}$ in diameter after $24 \mathrm{~h}$ ) and a $\beta$-haemolytic reaction on $5 \%$ sheep blood agar. Serogroup specificity was determined using a rapid agglutination test (Oxoid Streptococcal
Grouping Kit, Hampshire, UK). As $\beta$-haemolytic GCS and GGS causing human infections most frequently belong to the species SDSE, and the number of GCS was very low, a decision was made to treat them as one group (GCGS) for statistical purposes. Nonculturable bacteria were identified by $16 s r D N A$-PCR amplification from the sample material and subsequent sequencing, using primers and protocol as described previously [16]. Antimicrobial susceptibility testing of the invasive isolates had been performed according to Norwegian guidelines (http://www.unn.no/fag-og-forskning/arbeidsgruppen-for-antibiotikasporsmal-afa). However, routine testing for erythromycin-susceptibility and inducible clindamycin resistance was not performed prior to 2004. emm-typing of GAS, GCS and GGS was performed as described elsewhere [17]. GBS were serotyped by ImmuLex ${ }^{\text {tw }}$ Strep-B latex-agglutination (SSI Diagnostica, Denmark).

\section{Statistics}

Data were analysed using SPSS PASW STATISTICS (IBM SPSS Statistics for Windows, Version 21.0. Armonk, NY: IBM Corp). Categorical data were analysed using Chi square test or Fisher's exact test as appropriate. Non-parametric data were analysed using the MannWhitney $U$-test. For Tables 1 and 2 the $p$-values were adjusted for multiple comparisons ad modum HolmBonferroni. Population-data for the catchment area was obtained from Statistics Norway (http://www.ssb.no). Incidence rates were age and sex-adjusted to the 2001 Norwegian standard population. Time trends were evaluated by dividing the data into an early cohort (1999-2003) and a late cohort (2009-2013), and calculating mean incidence rate ratios (IRR) and a $p$-value. A twosided $p$-value $\leq 0.05$ was considered statistically significant.

\section{Results}

The 15-year survey period yielded 24 GAS, 45 GBS and 42 GCGS acute Osteoarticular Infections, representing 8,13 and $20 \%$ of the total count of invasive episodes, respectively. Two patients with GCGS acute OAI declined participation in the study. They are represented in the cumulative incidence, but omitted from all further analyses. One GBS and three SDSE were identified based on partial sequencing of $16 S$ rDNA gene from the four clinical samples that were culture negative. The proportions of the different subgroups of OAI are described in Table 1.

\section{Annual incidence and seasonal variation}

The cumulative incidence of GCGS OAI increased significantly from the first to the last 5-year period (IRR 5.7, $p=0.0003$ ), with annual incidence peaking at 1.9/100 000 in 2013 (Fig. 1). Conversely, the cumulative incidence of GAS and GBS OAI did not display 
Table 1 Demographics, comorbidity/risk factors and disease manifestations

\begin{tabular}{|c|c|c|c|c|c|c|c|c|c|}
\hline \multirow{2}{*}{ Demographics } & \multicolumn{2}{|c|}{ GAS $n=24$} & \multicolumn{2}{|c|}{ GBS $n=45$} & \multicolumn{2}{|c|}{ GCGS $n=40^{\mathrm{a}}$} & \multirow{2}{*}{\multicolumn{2}{|c|}{$\frac{\text { GAS vs GBS GAS vs GCGS }}{\text { Adjusted } p \text {-values }^{*}}$}} & \multirow[t]{2}{*}{ GBS vs GCGS } \\
\hline & & & & & & & & & \\
\hline Male sex & 15 & $63 \%$ & 21 & $47 \%$ & 25 & $63 \%$ & 0.62 & 1.0 & 0.95 \\
\hline Age Median (IQR) & 48 & $(8-72)$ & 70 & $(58-80)$ & 69 & $(49-81)$ & 0.007 & 0.049 & 1.0 \\
\hline \multicolumn{10}{|l|}{ Disease manifestation } \\
\hline $\mathrm{AOM}$ & 5 & $21 \%$ & 11 & $24 \%$ & 4 & $10 \%$ & 1.0 & 1.0 & 0.6 \\
\hline Vertebral AOM & 0 & & 7 & & 3 & & & & \\
\hline Peripheral AOM & 5 & & 4 & & 1 & & & & \\
\hline PJI & 5 & $21 \%$ & 22 & $49 \%$ & 15 & $38 \%$ & 0.148 & 1.0 & 1.0 \\
\hline Early PJI & 2 & & 6 & & 2 & & & & \\
\hline Delayed PJI & 3 & & 6 & & 5 & & & & \\
\hline Late PJI & 0 & & 10 & & 8 & & & & \\
\hline NJI & 14 & $58 \%$ & 12 & $27 \%$ & 21 & $53 \%$ & 0.049 & 1.0 & 0.11 \\
\hline \multicolumn{10}{|l|}{ Affected joint } \\
\hline Hip & 4 & $21 \%$ & 15 & $44 \%$ & 9 & $25 \%$ & & & \\
\hline Knee & 8 & $42 \%$ & 8 & $24 \%$ & 15 & $42 \%$ & & & \\
\hline Ankle & 2 & $11 \%$ & 1 & $3 \%$ & 3 & $8 \%$ & & & \\
\hline Shoulder & 0 & $0 \%$ & 1 & $3 \%$ & 4 & $11 \%$ & & & \\
\hline Other & 7 & $37 \%$ & 6 & $18 \%$ & 11 & $31 \%$ & & & \\
\hline Multiple joints & 0 & $0 \%$ & 5 & $15 \%$ & 1 & $3 \%$ & & & \\
\hline \multicolumn{10}{|l|}{ Microbiological cultures } \\
\hline Blood & 14 & $58 \%$ & 25 & $56 \%$ & 13 & $33 \%$ & & & \\
\hline Synovial Fluid & 14 & $58 \%$ & 11 & $24 \%$ & 23 & $58 \%$ & & & \\
\hline Tissue & 1 & $4 \%$ & 12 & $27 \%$ & 8 & $20 \%$ & & & \\
\hline Polymicrobial ${ }^{\text {b }}$ & 3 & $13 \%$ & 2 & $4 \%$ & 4 & $10 \%$ & & & \\
\hline \multicolumn{10}{|c|}{ Comorbidity and risk factors } \\
\hline Systemic risk factor & 10 & $42 \%$ & 37 & $82 \%$ & 23 & $58 \%$ & 0.005 & 1.0 & 0.1 \\
\hline Diabetes & 3 & $13 \%$ & 10 & $22 \%$ & 5 & $13 \%$ & & & \\
\hline Chr. Org. failure & 6 & $25 \%$ & 29 & $64 \%$ & 15 & $38 \%$ & & & \\
\hline Active malignancy & 1 & $4 \%$ & 8 & $18 \%$ & 5 & $13 \%$ & & & \\
\hline Immunosuppress & 1 & $4 \%$ & 2 & $4 \%$ & 4 & $10 \%$ & & & \\
\hline IVDU & 2 & $8 \%$ & 0 & $0 \%$ & 5 & $13 \%$ & & & \\
\hline Local risk factor & 10 & $42 \%$ & 29 & $64 \%$ & 29 & $73 \%$ & 0.21 & 0.084 & 1.0 \\
\hline Rheum. joint dis. & 0 & $0 \%$ & 4 & $9 \%$ & 6 & $15 \%$ & & & \\
\hline Prior SA & 5 & $21 \%$ & 5 & $11 \%$ & 10 & $25 \%$ & & & \\
\hline Prosthesis & 5 & $21 \%$ & 22 & $49 \%$ & 15 & $38 \%$ & & & \\
\hline Surg proc $<4$ weeks & 4 & $17 \%$ & 9 & $20 \%$ & 10 & $25 \%$ & & & \\
\hline Any risk factor & 15 & $63 \%$ & 41 & $91 \%$ & 37 & $93 \%$ & 0.046 & 0.048 & 1.0 \\
\hline
\end{tabular}

All data are presented as $\mathrm{n}(\%)$, except Age presented as median (inner quartile range (IQR)). Significant values are highlighted in bold

Abbreviations: GAS group A streptococci, GBS group B streptococci, GCGS group C and G streptococci, AOM acute osteomyelitis, PJl prosthetic joint infection,

$\mathrm{NJ}$ native joint infection, Chr. Org.failure chronic organ failure (according to medical records), IVDU active intravenous drug use, Rheum. Joint dis rheumatic joint disease, $S A$ septic arthritis, Surg proc $<4$ weeks surgical joint procedure performed within 4 weeks prior to admission

* The $p$-values are adjusted for multiple comparisons ad modum Holm-Bonferroni

a Two GCGS cases did not provide informed consent, and were excluded from the analyses

b Three GAS, one GBS and one GCGS blood culture positive cases had concomitant Staphylococcus aureus bacteraemia. One GBS and three GCGS tissue cultures also grew coagulase negative staphylococci 
Table 2 Clinical features, treatment and outcome

\begin{tabular}{|c|c|c|c|c|c|c|c|c|c|}
\hline \multirow{2}{*}{ Symptoms } & \multicolumn{2}{|c|}{ GAS $n=24$} & \multicolumn{2}{|c|}{ GBS $n=45$} & \multicolumn{2}{|c|}{ GCGS $n=40^{a}$} & \multirow{2}{*}{\multicolumn{2}{|c|}{$\frac{\text { GAS vs GBS GAS vs GCGS }}{\text { Adjusted } p \text {-values }^{*}}$}} & \multirow[t]{2}{*}{ GBS vs GCGS } \\
\hline & & & & & & & & & \\
\hline Duration days (IQR) & 1 & $(1-5)$ & 5 & $(2-13)$ & 3 & $(1-9)$ & 0.036 & 1.0 & 0.72 \\
\hline Fever & 21 & $88 \%$ & 30 & $67 \%$ & 23 & $58 \%$ & 0.63 & 0.09 & 1.0 \\
\hline \multicolumn{10}{|l|}{ Severity } \\
\hline SIRS & 18 & $75 \%$ & 23 & $51 \%$ & 24 & $60 \%$ & 0.48 & 1.0 & 1.0 \\
\hline STSS & 2 & $8 \%$ & 0 & $0 \%$ & 1 & $3 \%$ & 0.66 & 1.0 & 1.0 \\
\hline \multicolumn{10}{|l|}{ Surgical treatment } \\
\hline NJI & 14 & & 12 & & 21 & & & & \\
\hline Irrigation & 8 & $57 \%$ & 3 & $25 \%$ & 13 & $62 \%$ & & & \\
\hline Revision & 2 & $14 \%$ & 2 & $17 \%$ & 4 & $19 \%$ & & & \\
\hline Amputation & 2 & $14 \%$ & 0 & $0 \%$ & 2 & $10 \%$ & & & \\
\hline Antibiotics alone & 2 & $14 \%$ & 7 & $58 \%$ & 2 & $10 \%$ & & & \\
\hline PJI & 5 & & 22 & & 15 & & & & \\
\hline Revision & 5 & $100 \%$ & 9 & $41 \%$ & 7 & $47 \%$ & & & \\
\hline 1-stage & 0 & $0 \%$ & 2 & $9 \%$ & 1 & $7 \%$ & & & \\
\hline 2-stage & 0 & $0 \%$ & 5 & $23 \%$ & 3 & $20 \%$ & & & \\
\hline Amputation & 0 & $0 \%$ & 1 & $5 \%$ & 1 & $7 \%$ & & & \\
\hline Antibiotics alone & 0 & $0 \%$ & 5 & $23 \%$ & 3 & $20 \%$ & & & \\
\hline \multicolumn{10}{|l|}{ Treatment duration } \\
\hline NJl days (IQR) & 42 & $(32-52)$ & 38 & $(28-76)$ & 42 & $(34-56)$ & & & \\
\hline PJl days (IQR) & 90 & $(14-180)$ & 137 & $(76-361)$ & 91 & $(42-130)$ & & & \\
\hline AOM days (IQR) & 56 & $(42-56)$ & 60 & $(50-116)$ & 63 & $(51-81)$ & & & \\
\hline \multicolumn{10}{|l|}{ Outcome } \\
\hline Death <30 day & 2 & $8 \%$ & 1 & $2 \%$ & 1 & $3 \%$ & 1.0 & 1.0 & 1.0 \\
\hline Sequelae & 10 & $42 \%$ & 15 & $33 \%$ & 15 & $38 \%$ & 1.0 & 1.0 & 1.0 \\
\hline Amputation & 2 & $8 \%$ & 2 & $4 \%$ & 3 & $8 \%$ & 1.0 & 1.0 & 1.0 \\
\hline Relapse & 3 & $13 \%$ & 9 & $20 \%$ & 4 & $10 \%$ & 1.0 & 1.0 & 1.0 \\
\hline Chronic suppression & 0 & $0 \%$ & 5 & $11 \%$ & 1 & $3 \%$ & 0.8 & 1.0 & 1.0 \\
\hline
\end{tabular}

All data are presented as n (\%), except "Duration of symptoms" and "Treatment duration" presented as median number of days (Inner Quartile Range $(\mathrm{QQR})$ ). Significant values are highlighted in bold

Abbreviations: GAS group A streptococci, GBS group B streptococci, GCGS group C and G streptococci, SIRS systemic inflammatory response syndrome,

STSS streptococcal toxic shock syndrome, $N J$ native joint infection, $P J$ prosthetic joint infection, AOM acute osteomyelitis

* The $p$-values are adjusted for multiple comparisons ad modum Holm-Bonferroni

a Two GCGS cases did not provide informed consent, and were excluded from the analyses

any significant trends, and remained relatively stable at $0.5 / 100000$ (IRR $1.27, p=0.63$ ) and $0.8 / 100000$ (IRR 1.77, $p=0.14$ ), respectively.

All three groups exhibited seasonal variation, GAS peaking in the winter with $38 \%$ of the cases occurring from December to February, and only $13 \%$ during the summer-months of June through August. Differently, GBS and GCGS presented predominantly during summer (both $31 \%$ ), and less frequently in the winter (24 and $19 \%$, respectively).

\section{Demographics and comorbidity}

The demographics, risk factors and disease manifestations are shown in Table 1.
Both GBS and GCGS were significantly associated with the presence of comorbidity and risk factors; GBS clearly linked to systemic comorbidity, and GCGS predominantly associated with factors of local origin. Patients presenting with GBS or GCGS OAI were significantly older than the GAS OAI (corrected $p$-value $=0.007$ and $p=0.049$ ), and a male predominance was noted for GAS and GCGS. The age-difference is further delineated in Fig. 2, showing that the age-distribution for GBS and GCGS was skewed towards the elderly.

Clinical characteristics, treatment and outcome

Details on clinical severity, treatment and outcome are provided in Table 2. 


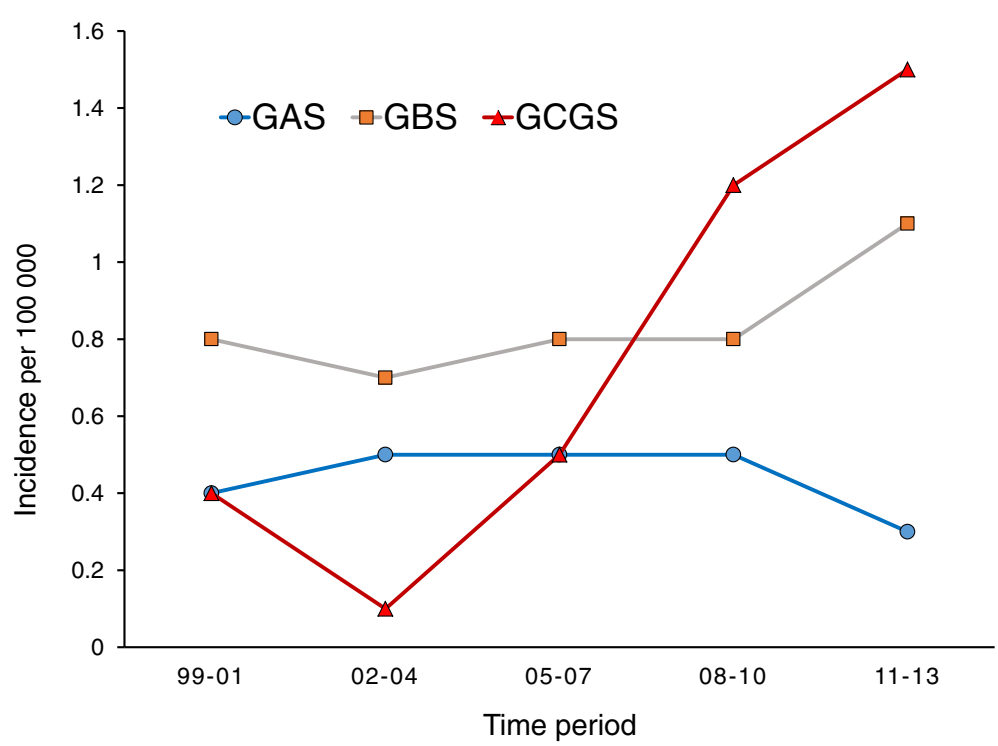

Fig. 1 Temporal trends of osteoarticular infections caused by GAS, GBS and GCGS in western Norway 1999-2013

OAI caused by GAS generally presented more acutely, and were frequently admitted to hospital within 1 day of disease onset. Furthermore, they were associated with increased severity and a worse outcome as compared to OAI caused by GBS and GCGS, although the finding did not reach clinical significance. Bacteraemia was present in 58 , 56 and $33 \%$ of GAS, GBS and GCGS-cases, respectively.

Most patients were treated with a combination of surgery and antibiotics. Penicillin was prescribed in $90 \%$ of the cases, whereas the rest were treated predominantly with Clindamycin or Cephalosporins. Between 10-20\% experienced recurring infections, the majority from relapse within 3 months after terminating antibiotic treatment. GBS was the pathogen most frequently implicated, and five patients with GBS OAI were eventually assigned to chronic suppressive antibiotic therapy. Recurrence was neither associated with antibiotic resistance patterns nor with specific emm/capsule-types. Although the overall mortality remained low, the morbidity was substantial. The proportion suffering from chronic sequelae ranged from $35-45 \%$, comprising predominantly arthritis and joint pain.

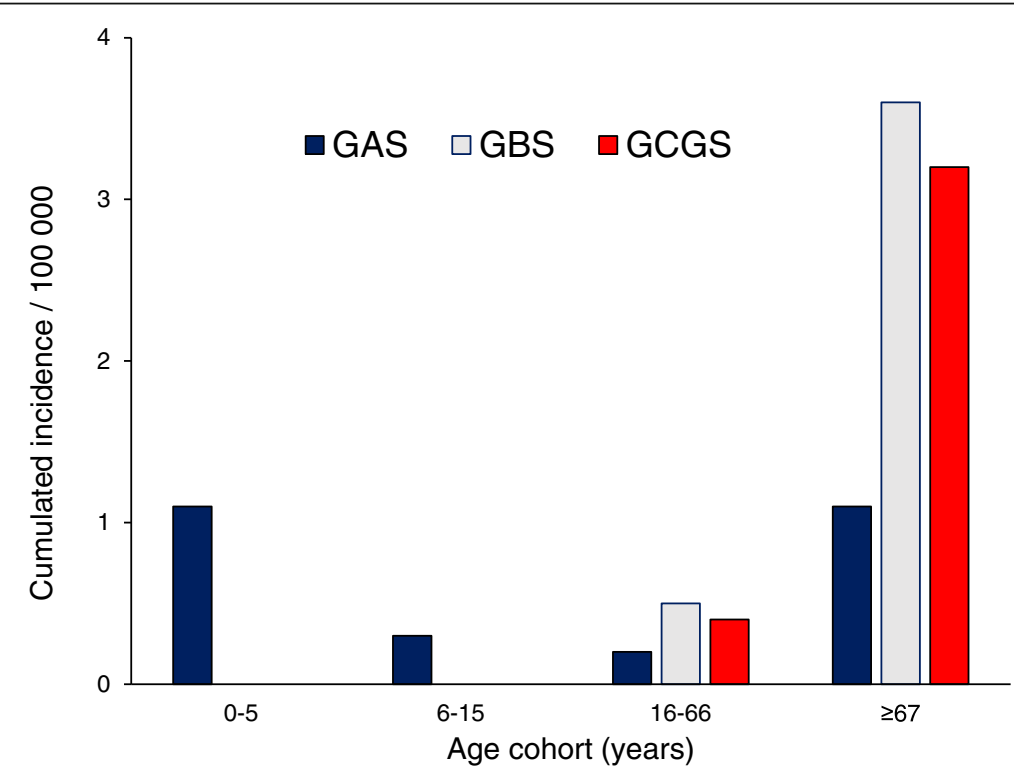

Fig. 2 Age group related cumulative incidence of osteoarticular infections caused by GAS, GBS and GCGS 
Molecular characteristics and antimicrobial susceptibility Altogether, 21 GAS, 30 GBS and 29 GCGS isolates were available for typing, revealing a diverse microbial population (Fig. 3). The major GAS emm-types were emm1 and emm3, and stG485 dominated among GCGS, although neither a clonal outbreak nor a temporal trend could be inferred. Among GBS, capsule-type III and V were most frequently identified.

All isolates were sensitive to penicillin. Resistance to erythromycin was detected in 9/41 (22\%) of GBS and 4/37 (11 \%) of GCGS, and resistance to clindamycin in 7/41 (17 \%) of GBS but in none of the GCGS. GAS were uniformly susceptible to erythromycin and clindamycin.

\section{Discussion}

Beta-haemolytic streptococci are an important cause of Osteoarticular infections (OAI), but their temporal trends have previously not been explored in detail. We found a highly significant increase in the GCGS OAI incidence during the 15-year survey period, the annual incidence peaking at 1.9/100 000 in 2013. To our knowledge, this has not been previously reported, although it is consistent with several epidemiological studies on invasive GCGS disease in general [11, 18, 19]. Conversely, the rates of GAS and GBS OAI remained relatively stable at $0.5 / 100000$ and $0.8 / 100000$, respectively. This contrasts the findings from a recent study, where a 3fold increase in the incidence of GBS bone-infections in the period 1995-2012 was documented [20]. However, that study also included chronic osteomyelitis, and $76 \%$ of the patients in their bone-infection cohort were diabetics. In line with our observations, though, they reported a stable incidence of $0.8 / 100000$ for GBS joint infections.

The increasing GCGS OAI incidence in our material could partly be due to increased microbial sampling or improved diagnostic procedures. However, one would expect this to be reflected also in the rates of GAS and GBS OAI, but these remained stationary. The ageing and increasingly comorbid population is probably of importance, but surprisingly did not affect the rates of GBS OAI, although GBS and GCGS both appear to preferentially target the elderly and the vulnerable patients. Acquisition of novel virulence factors enhancing invasive potential or conferring specific osteoarticular tissue tropism could be a contributing factor, and horizontal genetic transfer between streptococci has been inferred [21]. Experimental arthritis studies indicate a pivotal role for bacterial adhesins in the pathogenesis, but the knowledge on adhesins in GCGS is limited at present [22-24]. Osteoarticular infections are one of the major disease manifestations of GCGS, constituting approximately $20 \%$ of invasive disease, and further exploration of the molecular basis for their arthritogenicity is warranted [11].

We observed several species-associated clinical characteristics. GAS OAI tended to present more acutely, to produce a more severe clinical picture, and displayed the highest propensity to cause native joint infections. GBS and GCGS affected primarily the elderly, and were significantly associated with the presence of comorbidity and risk factors; GBS were clearly linked to systemic comorbidity, whereas GCGS were predominantly associated with local risk factors. Furthermore, GBS OAI were frequently polyarticular, and comprised the majority of the recurring infections. Our findings are consistent with those from previous publications [7-10].

The antibiotic treatment was concordant with established national guidelines regarding choice of antimicrobial agent and length of treatment. The surgical intervention, however, occasionally diverged from current standards [13]. Several PJIs were treated with curative intention with antibiotics alone, particularly in the earliest time-period. This probably had an impact on the rates of recurring infections and sequelae, although, GBS have been reported to be associated with high relapse rates in prosthetic joint infections compared to other pathogens [25].

Previously, emm4, emm 12 and emm 28 have been associated with infective arthritis [26-28]. We found a substantial molecular diversity among our isolates, and although these three emm-types were represented, they did not constitute the majority (Fig. 3). On the contrary,
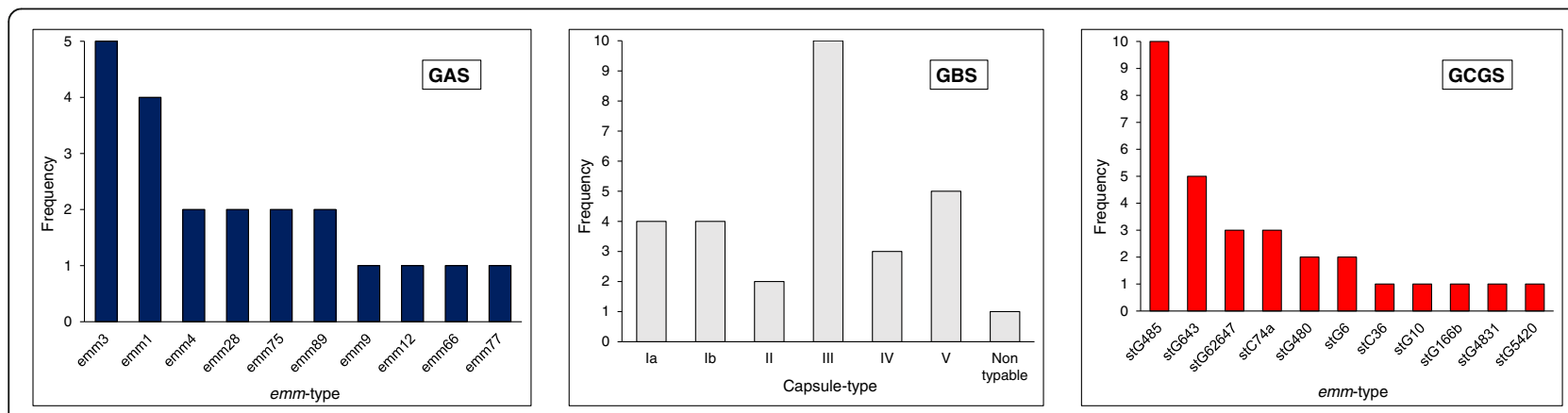

Fig. 3 Distribution of GAS and GCGS emm-types and GBS capsule-types 
the identified emm-types for both GAS and GCGS conform closely to the prevailing emm-types encountered in invasive disease in general during the study period [11]. Similarly, it is tempting to speculate that the OAI GBS serotypes likely reflects the most prevalent capsule types in the population, as previously documented in Norway [29].

The present study is limited by its retrospective design, increasing the risk of diagnostic misclassification. However, the microbe-specific emphasis of the study, along with a review of the local microbiological records, likely led to the inclusion of all cases of $\beta$-haemolytic streptococcal infection during the study period. Such an approach probably conforms more closely to the true disease burden than studies based on selected isolates submitted to National surveillance laboratories.

The total incidence and characteristics of OAI in our health region were unfortunately not available. Hence, a more comprehensive comparison of the clinical features of streptococcal OAI and OAI caused by other bacteria was not feasible.

Sepsis has recently been redefined, and the new classification should ideally have been applied to our sample material [30]. However, the use of the former sepsis definition based on SIRS-criteria permit a better comparison with existing literature on OAI and streptococcal disease in general. Furthermore, the retrospective design of the study hampered the categorization of the patients according to the new sepsis definition based on Sequential Organ Failure Assessment score results.

\section{Conclusions}

We found a significantly increasing incidence of GCGS Osteoarticular infections, whereas GAS and GBS OAI did not display any temporal trends. GCGS OAI were clearly associated with the presence of comorbidity and risk factors, predominantly of focal origin. With an increasing application of therapeutic and diagnostic bone and joint procedures, the rising trend of OAI caused by GCGS is likely to continue. Sustained epidemiological attentiveness to GCGS seems warranted.

\section{Abbreviations \\ AOM: Acute osteomyelitis; GAS: Group A streptococci; GBS: Group B streptococci; GCGS: Group C and group G streptococci; GCS: Group C streptococci; GGS: Group G streptococci; IQR: Inner quartile range; IRR: Incidence rate ratio; IVDU: Intravenous drug user; NJI: Native joint infection; OAl: Osteoarticular infections; PJl: Prosthetic joint infection; SDSE: Streptococcus dysgalactiae subspecies equisimilis; SIRS: Systemic inflammatory response syndrome; STSS: Streptococcal toxic shock syndrome}

\section{Acknowledgements}

We thank the staff at the microbiological department at Haukeland University Hospital for excellent technical assistance and for providing access to their laboratory facilities. Some of the results have been presented as an abstract/poster at the $26^{\text {th }}$ ECCMID conference in Amsterdam 10. April.2016.
Funding

No external funding was received.

\section{Availability of data and materials}

The data analysed in this study can be accessed by sending a request to the corresponding author, with approval of the institutional review boards.

\section{Authors' contributions}

BK and SS conceived the study. OO and HM contributed to the study design. OO extracted data, performed molecular analyses and drafted the manuscript. All authors read and approved the final manuscript.

\section{Competing interests}

The authors declare that they have no competing interests.

\section{Consent for publication}

Not applicable.

\section{Ethics approval and consent to participate}

The study underwent institutional ethics review and approval (2010/1406 Regional Ethics Committee West, Norway). Informed consent was obtained from participants.

\section{Author details}

${ }^{1}$ Department of Medicine, Haukeland University Hospital, 5021 Bergen, Norway. ${ }^{2}$ Department of Clinical Science, University of Bergen, Bergen, Norway. ${ }^{3}$ Department of Microbiology, Haukeland University Hospital, Bergen, Norway. ${ }^{4}$ Department of Medicine, Haraldsplass Deaconess Hospital, Bergen, Norway.

Received: 7 June 2016 Accepted: 27 September 2016

Published online: 04 October 2016

\section{References}

1. Geirsson AJ, Statkevicius S, Vikingsson A. Septic arthritis in Iceland 1990-2002: increasing incidence due to iatrogenic infections. Ann Rheum Dis. 2008:67(5):638-43.

2. Murillo O, Grau I, Lora-Tamayo J, Gomez-Junyent J, Ribera A, Tubau F, et al. The changing epidemiology of bacteraemic osteoarticular infections in the early 21st century. Clin Microbiol Infect. 2015 Mar;21 (3):254 e1-8.

3. Lim SY, Pannikath D, Nugent K. A retrospective study of septic arthritis in a tertiary hospital in West Texas with high rates of methicillin-resistant Staphylococcus aureus infection. Rheumatol Int. 2015;35(7):1251-6.

4. Kennedy N, Chambers ST, Nolan I, Gallagher K, Werno A, Browne M, et al. Native Joint Septic Arthritis: Epidemiology, Clinical Features, and Microbiological Causes in a New Zealand Population. J Rheumatol. 2015;42(12):2392-7

5. Dubost JJ, Couderc M, Tatar Z, Tournadre A, Lopez J, Mathieu S, et al. Three-decade trends in the distribution of organisms causing septic arthritis in native joints: single-center study of 374 cases. Joint Bone Spine. 2014;81(5):438-40.

6. Titecat M, Senneville E, Wallet F, Dezeque H, Migaud H, Courcol RJ, et al. Bacterial epidemiology of osteoarticular infections in a referent center: 10-year study. Orthop Traumatol Surg Res. 2013;99(6):653-8.

7. Deighton C. Beta haemolytic streptococci and musculoskeletal sepsis in adults. Ann Rheum Dis. 1993;52(6):483-7.

8. Osiri M, Akkasilpa S, Reinprayoon S, Deesomchok U Streptococcal arthritis in Thai adults: case series and review. J Med Assoc Thai. 1996;79(12):755-61.

9. Schattner A, Vosti KL. Bacterial arthritis due to beta-hemolytic streptococci of serogroups A, B, C, F, and G. Analysis of 23 cases and a review of the literature. Med (Baltimore). 1998;77(2):122-39.

10. Dubost JJ, Soubrier M, De Champs C, Ristori JM, Sauvezie B. Streptococcal septic arthritis in adults. A study of 55 cases with a literature review. Joint Bone Spine. 2004;71(4):303-11.

11. Oppegaard O, Mylvaganam H, Kittang BR. Beta-haemolytic group A, C and G streptococcal infections in Western Norway: a 15-year retrospective survey. Clin Microbiol Infect. 2015;21(2):171-8.

12. Newman JH. Review of septic arthritis throughout the antibiotic era. Ann Rheum Dis. 1976;35(3):198-205.

13. Osmon DR, Berbari EF, Berendt AR, Lew D, Zimmerli W, Steckelberg JM, et al. Diagnosis and management of prosthetic joint infection: clinical practice 
guidelines by the Infectious Diseases Society of America. Clin Infect Dis. 2013;56(1):e1-e25.

14. Levy MM, Fink MP, Marshall JC, Abraham E, Angus D, Cook D, et al. 2001 SCCM/ESICM/ACCP/ATS/SIS International Sepsis Definitions Conference. Intensive Care Med. 2003;29(4):530-8.

15. Defining the group A streptococcal toxic shock syndrome. Rationale and consensus definition. The Working Group on Severe Streptococcal Infections. JAMA. 1993;269(3):390-1.

16. Kommedal O, Kvello K, Skjastad R, Langeland N, Wiker HG. Direct $16 \mathrm{~S}$ rRNA gene sequencing from clinical specimens, with special focus on polybacterial samples and interpretation of mixed DNA chromatograms. J Clin Microbiol. 2009;47(11):3562-8.

17. Kittang BR, Langeland N, Mylvaganam H. Distribution of emm types and subtypes among noninvasive group A, C and $\mathrm{G}$ streptococcal isolates in western Norway. APMIS. 2008;116(6):457-64.

18. Lambertsen LM, Ingels H, Schonheyder HC, Hoffmann S. Danish Streptococcal Surveillance Collaboration G. Nationwide laboratory-based surveillance of invasive beta-haemolytic streptococci in Denmark from 2005 to 2011. Clin Microbiol Infect. 2014;20(4):O216-23.

19. Harris P, Siew DA, Proud M, Buettner P, Norton R. Bacteraemia caused by beta-haemolytic streptococci in North Queensland: changing trends over a 14-year period. Clin Microbiol Infect. 2011;17(8):1216-22.

20. Smith EM, Khan MA, Reingold A, Watt JP. Group B streptococcus infections of soft tissue and bone in California adults, 1995-2012. Epidemiol Infect. 2015;143(15):3343-50.

21. Choi SC, Rasmussen MD, Hubisz MJ, Gronau I, Stanhope MJ, Siepel A. Replacing and additive horizontal gene transfer in Streptococcus. Mol Biol Evol. 2012;29(11):3309-20.

22. Colavite PM, Sartori A. Septic arthritis: immunopathogenesis, experimental models and therapy. J Venom Anim Toxins Incl Trop Dis. 2014;20:19.

23. Jonsson IM, Pietrocola G, Speziale P, Verdrengh M, Tarkowski A. Role of fibrinogen-binding adhesin expression in septic arthritis and septicemia caused by Streptococcus agalactiae. J Infect Dis. 2005;192(8):1456-64.

24. Kreikemeyer B, Nakata M, Oehmcke S, Gschwendtner C, Normann J, Podbielski A. Streptococcus pyogenes collagen type I-binding Cpa surface protein. Expression profile, binding characteristics, biological functions, and potential clinical impact. J Biol Chem. 2005:280(39):33228-39.

25. Zeller V, Lavigne M, Biau D, Leclerc P, Ziza JM, Mamoudy P, et al. Outcome of group B streptococcal prosthetic hip infections compared to that of other bacterial infections. Joint Bone Spine. 2009;76(5):491-6.

26. Imohl M, Reinert RR, Ocklenburg C, van der Linden M. Epidemiology of invasive Streptococcus pyogenes disease in Germany during 2003-2007. FEMS Immunol Med Microbiol. 2010;58(3):389-96.

27. Plainvert C, Doloy A, Loubinoux J, Lepoutre A, Collobert G, Touak G, et al. Invasive group A streptococcal infections in adults, France (2006-2010). Clin Microbiol Infect. 2012;18(7):702-10.

28. Vlaminckx BJ, Mascini EM, Schellekens J, Schouls LM, Paauw A, Fluit AC, et al. Site-specific manifestations of invasive group a streptococcal disease: type distribution and corresponding patterns of virulence determinants. J Clin Microbiol. 2003:41(11):4941-9.

29. Bergseng H, Rygg M, Bevanger L, Bergh K. Invasive group B streptococcus (GBS) disease in Norway 1996-2006. Eur J Clin Microbiol Infect Dis. 2008;27(12):1193-9.

30. Singer M, Deutschman CS, Seymour CW, Shankar-Hari M, Annane D, Bauer M, et al. The Third International Consensus Definitions for Sepsis and Septic Shock (Sepsis-3). JAMA. 2016;315(8):801-10.

\section{Submit your next manuscript to BioMed Central and we will help you at every step:}

- We accept pre-submission inquiries

- Our selector tool helps you to find the most relevant journal

- We provide round the clock customer support

- Convenient online submission

- Thorough peer review

- Inclusion in PubMed and all major indexing services

- Maximum visibility for your research

Submit your manuscript at www.biomedcentral.com/submit
C Biomed Central 